\title{
THE IMPACT OF ANXIETY SENSITIVITY ON SUICIDALITY IN ADOLESCENTS WITH MAJOR DEPRESSIVE DISORDER
}

\author{
Ayhan Bilgiç, Savaş Yılmaz \& Sabri Hergüner \\ Necmettin Erbakan University, Meram School of Medicine, Department of Child and Adolescent Psychiatry \\ Meram / Konya, Turkey
}

received: 2.6.2016;

revised: 25.11.2016;

accepted: 9.1.2017

\begin{abstract}
SUMMARY
Background: Few studies have examined the link between anxiety sensitivity (AS) and suicidality among adolescents with major depressive disorder; thus, there is a need to explore this relationship in order to fill the research gap. The aim of this cross-sectional study was to examine the association among AS and suicidal behaviour while taking into account the effects of potential third variables such as the presence of anxiety disorders and hopelessness in adolescents with major depressive disorder.

Subjects and methods: Participants ( $n=101)$ completed self-report measures of depressive symptoms, AS, hopelessness and suicidal ideation severity. The presence of anxiety disorders and suicide attempt history were determined with psychiatric interviews. The association between psychiatric variables was evaluated using structural equation modelling.

Results: Results showed that AS cognitive concerns had a positive effect on suicidal ideation mediated by the severity of depression. AS physical concerns had a negative direct effect on suicidal ideation, but there was also a positive relationship among these variables through hopelessness, thus, the total effect of AS physical concerns was not significant. There were also positive relationships between hopelessness and suicidal ideation, and the presence of anxiety disorders to suicide attempt history.

Conclusions: The results of this study suggest that higher AS cognitive concerns may be a risk factor for suicidality through the severity of depression in adolescents who are diagnosed with major depressive disorder.
\end{abstract}

Key words: anxiety sensitivity - anxiety - depression - suicide - adolescent

$* * * * *$

\section{INTRODUCTION}

Depression is strongly associated with suicidal thoughts and behaviours in adolescents (Ghaziuddin et al. 2000, Gould et al. 1998). It typically co-occurs with other psychiatric conditions and is related to cognitive, behavioural and developmental factors (Davidson et al. 2002). However, few studies have investigated the relationships among dimensions of adolescent depression and suicidality. Understanding the risk factors for suicidal behaviours in these patients is critical for the development of preventive suicide strategies. Thus, we should develop more parsimonious models for clarifying the association between depression and suicidality in adolescents.

The coexistence rate of anxiety disorders is high in individuals with depression during adolescence (Mathew et al. 2011, Maughan et al. 2013). Although anxiety disorders have not been traditionally viewed as independent risk factors for suicidal acts, research conducted over the past several decades suggests that specific anxiety disorders are independently associated with suicide (Beautrais et al. 2006, Foley et al. 2006, Ghaziuddin et al. 2000, Vanderwerker et al. 2007). Moreover, previous studies have found that a baseline lifetime history of anxiety disorders, including panic disorder, generalized anxiety disorder, social phobia, agoraphobia, post-traumatic stress disorders (PTSDs), and specific phobias, are associated with an increased risk of new onset suicide attempts after adjusting for comorbid mood disorders (Sareen et al. 2005). Therefore, a better understanding of the interplay between anxiety and suicidality may have valuable clinical implications for the management of suicidal acts in depressive adolescents.

Anxiety sensitivity (AS) refers to a fear of anxietyrelated arousal sensations and may represent the constitutionally based sensitivity of individuals to anxiety and anxiety symptoms (Reiss et al. 1986). AS is associated with the development of a range of psychopathologies that include anxiety disorders and depression (Schmidt et al. 2006). Although factor-analytical investigations have yielded controversial results, evidence from previous studies suggests that AS can be represented by three factor structures: physical, cognitive and social subscales (Essau et al. 2010, McLaughlin et al. 2007). These factors represent fear of anxiety-related bodily sensations, fear of cognitive dyscontrol and fear of externally observable anxiety symptoms, respectively. Previous studies have examined the relationship between AS and suicide in adults. Schmidt et al. found that a higher AS for cognitive concerns was associated with increased vulnerability for suicide ideation in adults who had panic disorder (Schmidt et al. 2001). Moreover, a study in a diverse group of clinical outpatients showed that total AS and AS cognitive concerns were positively related to suicidal ideation and suicide attempts, and that AS social concerns were positively associated with suicide attempts (Capron et al. 2012a). Another study by the same authors found that higher AS 
cognitive scores and lower AS physical scores were positive predictors of suicide attempts in an outpatient sample with PTSD symptomatology (Capron et al. 2012b). A recent longitudinal adolescent study investigated the link between AS and suicidal behaviour and provided empirical support for a relationship between AS cognitive concerns and suicidality (Capron et al. 2015).

Several hypotheses have been proposed to explain the multifaceted relationship between the AS subfactors and suicidality (Capron et al. 2012a, Katz et al. 2011). From a theoretical perspective, higher AS cognitive concerns, which refer to the fear of loss of cognitive control in the context of stress and anxiety, cause catastrophic cognition in individuals who are vulnerable to depression (Capron et al. 2012a). Catastrophic thinking activates the limbic-autonomic arousal system and activates a positive feedback loop, which becomes amplified over time (Katz et al. 2011). Eventually, individuals with elevated AS cognitive concerns will develop strong suicidal ideations and may attempt suicide to stop the escalating stress (Capron et al. 2012a). Conversely, AS physical concerns represent the fear of bodily sensations, including pain; thus, according to the interpersonal-psychological theory of suicide (Joiner 2005, Ocañez et al. 2010), individuals with elevated AS physical concerns are likely to avoid the self-inflicted pain that may be caused by suicide attempt. A previous study posited that the interpersonal-psychological theory of suicide also explained the positive association between AS social concerns and suicide because these have a close relationship with a lack of belongingness (Capron et al. 2012a).

Given that high AS is a risk factor for the development of anxiety disorders, it is important to control for anxiety disorders in the assessment of the AS impact on suicidality, as it may be that AS increases the risk of suicide through its role in the development of anxiety disorders. However, few previous studies that have examined the relationship between AS and suicidality controlled for the presence of anxiety disorders or the severity of anxiety; of those that did, the investigations were confined to the relationship between AS and suicidal ideation (Capron et al. 2012b, Capron et al. 2012c). Hopelessness is also among the important causes of suicidality; therefore, taking into account its impact on suicidal behaviours may be beneficial. The present study investigated the impact of AS dimensions on current suicidal ideation severity and the history of suicide attempts in adolescents with major depressive disorder (MDD). This was done after adjusting for the influence of other potential powerful predictors of suicidality, including the presence of anxiety disorders, severity of depression, and hopelessness.

\section{SUBJECTS AND METHODS}

\section{Participants}

The study sample consisted of 101 adolescents (1218 years old) with MDD who attended the Child and
Adolescent Psychiatry Clinics of the Meram Faculty of Medicine. All participants were outpatients and MDD was diagnosed by experienced child and adolescent psychiatrists according to the DSM-IV criteria using the Kiddie Schedule for Affective Disorders and Schizophrenia-Present and Lifetime version (K-SADS-PL) (Kaufman et al. 1997). The exclusion criteria included the presence of a major physical/neurological illness (e.g., cardiovascular disorders and epilepsy), schizophrenia, bipolar disorders, pervasive developmental disorders, and substance abuse/dependence (except smoking). Furthermore, patients who had taken any psychopharmacological agents within the previous three months were excluded. Two parents refused to participate in the study, and 14 children were excluded according to the exclusion/inclusion criteria.

\section{Measures/Instruments}

Sociodemographic and Medical History: A standardized form was used to obtain participant demographic and clinical data including age and sex. The history of attempted suicide was determined by asking participants if they had ever attempted suicide and responses were categorized as "no previous attempt" or "previous attempt" (Schmidt et al. 2001).

\section{K-SADS-PL}

The K-SADS-PL is a widely used semi-structured diagnostic interview tool used to assess current and past episodes of child and adolescent psychiatric disorders. The Turkish version of the K-SADS-PL has good testretest and inter-rater reliability (Kaufman et al. 1997, Gokler et al. 2004). We used the K-SADS-PL to diagnose MDD and anxiety disorders.

\section{Children's Depression Inventory (CDI)}

The CDI is a self-report depression scale for children between the ages of 6 and 17 years (Kovacs 1985). The CDI is comprised of 27 items scored as 0,1 or 2 according to the severity of the symptoms in the previous two weeks. Oy (1991) has assessed the reliability and validity of the scale for the Turkish population.

\section{The Suicidal Ideation and the Hopelessness Subscales} of the Suicide Probability Scale (SPS-SIS and SPS-H)

The SPS-SIS and the SPS-H are subscales of the self-report Suicide Probability Scale, which evaluate the severity of suicidal thoughts and hopelessness in adolescents and adults (Cull \& Gill 1998, Eskin 1999). Individuals are asked to rate the frequency of their subjective experiences and past behaviours using a four-point Likert scale ranging from "1 (none)" to "4 (all of the time)". Higher points indicate higher suicide risks and hopelessness.

\section{The Childhood Anxiety Sensitivity Index (CASI)}

The CASI is a self-report instrument developed to assess AS in children (Silverman et al. 1999). The CASI includes 18 items that measure fear and catastrophic interpretations of anxiety-related sensations using a three-point Likert-type scale ranging from "1 (not at 
all)" to "3 (very much)". The CASI has good internal consistency and test-retest reliability in clinical and nonclinical samples, it also has good concurrent and predictive validity (Lambert et al. 2004, McLaughlin et al. 2007). Moreover, this instrument has incremental validity over anxiety symptoms. Although the results of factor analytic investigations of the CASI have been controversial, recent evidence suggests that AS can be represented by three factor structures: physical, cognitive and social subscales. These factors represent a fear of anxiety-related bodily sensations, a fear of loss of cognitive control and a fear of externally observable anxiety symptoms, respectively. The reliability of the Turkish version was assessed by Yılmaz and Kılıç (2014) in Turkish school children.

\section{Procedures}

Our study was approved by the Institutional Ethical Committee. The patients and parents who agreed to participate in the study were provided with patient information sheets that outlined the research protocol, and all subjects provided written and oral informed consent. All subjects were interviewed by a child and adolescent psychiatrist using the K-SADS-PL, and psychiatric diagnoses were made according to DSM-IVTR criteria. The sociodemographic and medical history forms were completed by the psychiatrists. The patients were then asked to complete the CDI, SPS-SIS, SPS-H, and CASI.

\section{Statistical Analysis}

Data analysis was performed using SPSS 20.0AMOS statistical software (SPSS Inc., Chicago, IL, US). Pearson's or Spearman correlation coefficients were calculated among the variables. To determine the relationship between psychiatric variables, structural equation modelling (SEM) using bootstrapping was performed. In this study, to evaluate the goodness of fit of a model the relative/normed chi-square $\left(\chi^{2} / \mathrm{df}\right)$, the incremental fit index (IFI), the comparative fit index (CFI), and the root mean square error of approximation
(RMSEA) statistics were used. Although there is no consensus regarding an acceptable ratio for $\chi^{2} / \mathrm{df}$, authors usually recommend it to be under 2.0 (Tabachnick \& Fidell 2007). For RMSEA, the lower limit is close to 0 whereas the upper limit should be $<0.08$ for a well-designed model. For IFI and CFI, a cutoff criterion of $\geq 0.95$ has been recommended, but $\geq 0.90$ has also been considered acceptable ( $\mathrm{Hu} \&$ Bentler 1999, Tabachnick \& Fidell 2007). All of these parameters would be fulfilled for an acceptable model. Estimates of path coefficient in SEM analyses reflected the strength of the path between the predictor and outcome variables and were calculated by standardized regression coefficients (i.e., $\beta$ value). As well as the direct paths, the significance of indirect paths among variables was also evaluated. A two-tailed p-value of $<0.05$ was deemed to indicate statistical significance for all of the analyses.

\section{RESULTS}

The study sample consisted of $25(24.8 \%)$ males and $76(75.2 \%)$ females with a mean age of $15.4 \pm 1.2$ years (range: 12-18 years). Twenty-eight of the participants $(28.3 \%)$ had a history of attempted suicide. The mean duration from suicide attempt to study baseline was 4.8 \pm 7.6 months. According to the K-SADS-PL, the frequency of having at least one anxiety disorder (with the exception specific phobias) was $34.7 \%$, with the most frequent anxiety disorders being generalized anxiety disorder (18.8\%), social phobia (12.9\%), obsessive compulsive disorder $(8.9 \%)$, panic disorder (5.0\%) and PTSD (4.0\%).

The bivariate correlations between the variables are shown in Table 1. The CASI total score was positively correlated with the CDI score, SPS-H and the presence of anxiety disorders. The CASI cognitive subscale was positively correlated with the CDI, SPS-SIS, SPS-H scores and the presence of anxiety disorders; the physical subscale was associated with the presence of anxiety disorders and SPS-H; and the social subscale was not associated with any of the measures.

Table 1. Intercorrelations among study variables

\begin{tabular}{|c|c|c|c|c|c|c|c|c|c|}
\hline & $\begin{array}{c}\text { Mean } \pm \\
\text { SD/frequency }\end{array}$ & 2 & 3 & 4 & 5 & 6 & 7 & 8 & 9 \\
\hline CASI total & $35.53 \pm 6.49$ & $0.93 * * *$ & $0.50 * * *$ & 0.06 & $0.21 *$ & 0.04 & $0.32 * *$ & $0.24 *$ & -0.14 \\
\hline CASI physical & $23.50 \pm 5.80$ & & $0.23 *$ & -0.14 & 0.13 & -0.01 & $0.22 *$ & $0.22 *$ & -0.12 \\
\hline CASI cognitive & $5.62 \pm 1.99$ & & & -0.07 & $0.42 * * *$ & $0.22 *$ & $0.46^{* * *}$ & $0.23 *$ & 0.01 \\
\hline CASI social & $6.42 \pm 1.38$ & & & & -0.19 & -0.11 & -0.12 & -0.10 & -0.10 \\
\hline CDI & $26.4 \pm 8.52$ & & & & & $0.64 * * *$ & $0.62 * * *$ & 0.19 & $0.30 * *$ \\
\hline SPS-SIS & $18.20 \pm 6.57$ & & & & & & $0.63 * * *$ & 0.11 & $0.42 * * *$ \\
\hline SPS-H & $32.42 \pm 5.53$ & & & & & & & $0.21 *$ & $0.23^{*}$ \\
\hline $\begin{array}{l}\text { Presence of } \\
\text { anxiety disorder }\end{array}$ & $34.7 \%$ & & & & & & & & $0.22 *$ \\
\hline $\begin{array}{l}\text { History of } \\
\text { suicide attempts }\end{array}$ & $27.2 \%$ & & & & & & & & \\
\hline
\end{tabular}

CDI, Child Depression Inventory; CASI, Childhood Anxiety Sensitivity Index, SPS-SIS, Suicide Probability Scale-

Suicidality Ideation Subscale; SPS-H, Suicide Probability Scale- Hopelessness Subscale; ${ }^{*} \mathrm{p}<0.05,{ }^{* *} \mathrm{p}<0.01,{ }^{* * *} \mathrm{p}<0.001$ 
Table 2. Statistics of indirect and total effects in the SEM model

\begin{tabular}{lcccc} 
& \multicolumn{2}{c}{ Indirect Effect } & Total Effect \\
& Standardized estimate & SE & Standardized estimate & SE \\
\hline CASI cognitive $\rightarrow$ SPS-H & $0.245^{* *}$ & 0.164 & $0.245^{* *}$ & 0.164 \\
CASI cognitive $\rightarrow$ SPS-SIS & $0.272^{* *}$ & 0.181 & $0.272^{* *}$ & 0.181 \\
CASI cognitive $\rightarrow$ Suicide attempt & $0.114^{* *}$ & 0.007 & $0.114^{* *}$ & 0.007 \\
CASI physical $\rightarrow$ SPS-SIS & $0.069^{*}$ & 0.039 & -0.083 & 0.100 \\
CASI physical $\rightarrow$ Suicide attempt & 0.008 & 0.004 & 0.008 & 0.004 \\
CDI $\rightarrow$ SPS-SIS & $0.228^{* *}$ & 0.045 & $0.644^{* *}$ & 0.055 \\
CDI $\rightarrow$ Suicide attempt & $0.271^{* *}$ & 0.003 & $0.271^{* *}$ & 0.003 \\
SPS-H $\rightarrow$ Suicide attempt & $0.165^{* *}$ & 0.004 & $0.165^{* *}$ & 0.004 \\
\hline
\end{tabular}

CDI: Child Depression Inventory; CASI: Childhood Anxiety Sensitivity Index; SPS-SIS: Suicide Probability Scale -

Suicidality Ideation Subscale; SPS-H: Suicide Probability Scale - Hopelessness Subscale; SE, Standard Error;

$* \mathrm{p}<0.05, \quad * * \mathrm{p}<0.01, \quad * * * \mathrm{p}<0.001$

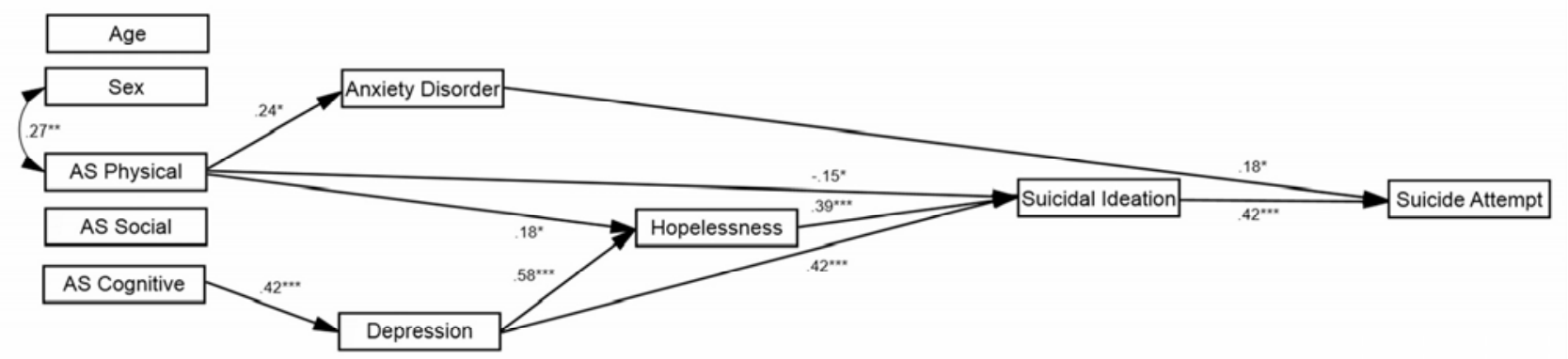

AS, anxiety sensitivity; $\chi^{2}=52.144 ; \quad \mathrm{df}=35 ; \quad \chi^{2} / \mathrm{df}=1.490 ;$ root mean square error of approximation $(\mathrm{RMSEA})=0.070$; incremental fit index $(\mathrm{IFI})=0.912 ; \quad$ comparative fit index $(\mathrm{CFI})=0.907 ; \mathrm{p}=0.031,{ }^{*} \mathrm{p}<0.05,{ }^{* *} \mathrm{p}<0.01,{ }^{* * *} \mathrm{p}<0.001$

Figure 1. Structural model for the relationships of depression severity, anxiety disorders, anxiety sensitivity, hopelessness and suicidal behaviors

SEM was used to identify the relationship of psychiatric variables that included the SPS-SIS, SPS-H, CDI, CASI subscales and diagnosis of an anxiety disorder with suicidal ideation measured using the SPSSIS and suicide attempt history. Due to their potential impact on variables, age and sex were controlled for in this analysis. Overall, the model fit the data satisfactorily $\quad\left(p=0.031, \quad \chi^{2} / \mathrm{df}=1.490, \quad \mathrm{RMSEA}=0.070\right.$, $\mathrm{IFI}=0.912$, and $\mathrm{CFI}=0.907)$. The standardized path coefficients for the model are presented in Figure 1 (direct effects). The results revealed that the CASI cognitive scores had a positive regression relationship with depressive symptoms $(\beta=0.422, p<0.001)$, and in turn, depressive symptoms had positive regression relationships with SPS-SIS $(\beta=0.416, p>0.001)$ and the SPS-H $(\beta=0.581, \mathrm{p}<0.001)$. The CASI physical scores were positively related to the SPS-H $(\beta=0.392, p=0.017)$ and the diagnosis of an anxiety disorder $(\beta=0.237$, $\mathrm{p}=0.015)$, whereas they were negatively related to the SPS-SIS $(\beta=-0.152, p=0.032)$. There were also positive relationships among the SPS-H to SPS-SIS $(\beta=0.392$, $\mathrm{p}<0.001)$ and SPS-SIS to suicide attempt history $(\beta=0.420, p<0.001)$. Regarding the diagnosis of an anxiety disorder, it alone was significantly associated with the suicide attempt history $(\beta=0.180, \mathrm{p}=0.044)$.

Several significant indirect effects were also identified. The CASI cognitive subscale had indirect relationships with SPS-H $(\beta=0.245, p=0.005)$, SPS-SIS $(\beta=0.272$, $\mathrm{p}=0.003)$, and suicide attempt history $(\beta=0.114$, $\mathrm{p}=0.002)$. The CASI physical subscale had an indirect relationship with SPS-SIS $(\beta=0.069, p=0.026)$. The depression severity had indirect relationships with SPSSIS $(\beta=0.228, p=0.005)$ and suicide attempt history $(\beta=0.271, p=0.002)$. The SPS-H had an indirect relationship with suicide attempt history $(\beta=0.165 \mathrm{p}=0.003)$. Indirect and total effects from SEM relating to suicidality are given in Table 2 .

\section{DISCUSSION}

Our results provide evidence for a potential increased effect of AS cognitive concerns on suicidality mediated by the severity of depression among adolescents diagnosed with MDD. This study also showed a positive relationship with AS physical concerns to suicidal ideation. However, a negative indirect relationship was also detected among AS physical concerns to suicidal ideation through hopelessness; therefore, the total effect of AS physical concerns did not remain significant. With regard to anxiety disorders, our findings suggest that the presence of anxiety disorders may have a direct relationship with suicide attempts.

Although agreement is not universal, most studies have found that AS cognitive concerns are a risk factor for previous suicide attempts in adults (Capron 2012a, Capron 2012b, Capron 2013). Some of these studies did 
not control for potential confounders such as anxiety disorders and depression, but others found unique predictor effects of AS cognitive concerns on suicidal ideation after accounting for these variables (Lamis \& Jahn 2013, Schmidt et al. 2001). In light of these findings, Capron and colleagues (2013) proposed a depression distress amplification model to explain the mechanism underlying the effect of AS on suicidal behaviours. According to this model, higher AS cognitive concerns represent catastrophic cognition about anxiety experiences, thus, amplifying the existing dysphoria and suicidal ideation in individuals with psychiatric disorders. Therefore, an individual with high AS cognitive concerns may be at risk for attempted suicide. In line with this model, a recent study by the same authors have found that baseline AS cognitive concerns have a predictive effect on suicidal ideation two years later in adolescents, independent from baseline suicidal ideation and depression (Capron et al. 2015). The authors have also shown that increased suicidal ideation is the result of interactions between AS cognitive concerns and depressive symptoms, not anxiety symptoms. Our finding of AS cognitive concerns on suicidal ideation through depression symptoms is consistent with this report and supports the depression distress amplification model (Capron et al. 2013).

There is data suggesting a positive relationship among AS social concerns and previous suicide attempts in adults (Capron et al. 2012a). In contrast, higher AS physical concerns have been reported to protect against suicidal acts (Capron et al. 2012b). A similar relationship between AS social and physical concerns and suicidal ideation has also been reported (Capron et al. 2012a, Capron et al. 2012b, Capron et al. 2013, Lamis \& Jahn 2013). However, the previously discussed longitudinal adolescent study did not confirm an association of AS physical and social concerns with suicidality (Capron et al. 2015). Confirming that report, the present study did not find any direct or indirect association between AS physical and AS social concerns with suicidal behaviours. Because AS is a risk factor for the development of anxiety disorders that increase the risk of suicidal behaviours, the lack of control over anxiety disorders or anxiety severity would be responsible for divergent results of past studies.

Apart from the association between AS and suicidality, the present study has also provided interesting data concerning the relationships among anxiety disorders and suicidal behaviours. Our results demonstrate that the presence of an anxiety disorder is related with suicide attempts independent from the severity of depression. Some studies have supported this opinion by observing the importance of the anxiety disorders in suicide risk. For instance, Brière et al. (2015) observed that adolescent suicide attempts were predictive of anxiety disorders but not depression in adults. Beghi et al. (2010) indicated the increased risk of anxiety disorders in adolescents who had suicidal self-harm above and beyond depression. A recent follow-up study showed a link between anxiety symptoms to new-onset suicidal ideation after controlling for the lifetime history of suicide attempts, baseline severity of suicide risk and depression (Baek et al. 2015). Until now, mechanisms of the links between anxiety disorders to suicidality and its differences and similarities with the links between depression and suicidality have not been fully elucidated. Given the unique longitudinal relationship among AS cognitive concerns and suicidal ideation through depression but not anxiety symptoms, Capron et al. (2015) suggest AS cognitive concerns may be an important construct to explain the association between suicidality and depression but not anxiety disorders. Therefore, higher comorbidity of anxiety disorders and depression among adolescents with suicidal behaviours may not solely account for simple comorbidity, but rather, rely on specific mechanisms (Capron et al. 2015). Our findings, which showed different links of depression severity and anxiety disorders with suicidal behaviours, may be considered to be in line with this view and may point out the specific effects of depression and anxiety on suicidality in adolescents.

The results of our study should be interpreted in light of its limitations. First, our samples were selected from patients attending an outpatient clinic and, as such, may not reflect the general population because of biases associated with treatment-seeking individuals. Second, our small sample size did not allow us to analyse the relationships between different anxiety disorder types and suicidality. Finally, the item used to assess history of suicide attempts did not allow us to assess the severity of the attempt, and we did not differentiate between past and current attempts in the "previous attempt" category. Measurement of the severity of suicide attempts and the separate evaluations of current and past suicide attempts may help to further clarify the relationship between AS and attempted suicide. The cross-sectional nature of our study and the preponderance of females in our sample also may have affected our findings.

\section{CONCLUSIONS}

Limitations notwithstanding, our findings suggest that AS cognitive concerns may have an amplificative effect on suicidal behaviours via a mediating effect of depressive symptoms, whereas AS physical and social concerns are not associated with suicidality in adolescents. Furthermore, our findings suggest that the presence of anxiety disorders may be a risk factor for suicidal attempts and the effect of anxiety disorders and depression on suicidality may take place under different specific mechanisms. Though this notion must be further elaborated, our data may be useful for understanding suicidal behaviours in adolescents with MDD and may offer new perspectives or directions for future research. 


\section{Acknowledgements: None.}

Conflict of interest: None to declare.

\section{Contribution of individual authors:}

Ayhan Bilgiç, Savaş Yılmaz and Sabri Hergüner were responsible for the study conception and acquisition of data.

Ayhan Bilgiç and Savaş Yılmaz provided statistical expertise.

Ayhan Bilgiç. were responsible for the literature searches and design of the manuscript.

\section{References}

1. Beautrais A, Wells J, McGee M, Oakley Browne M, Oakley Browne MA, New Zealand Mental Health Survey Research, T: Suicidal behaviour in Te Rau Hinengaro: the New Zealand Mental Health Survey. Aust $N Z J$ Psychiatry 2006; 40:896-904.

2. Baek JH, Heo JY, Fava M, Mischoulon D, Nierenberg A, Hong JP, Roh S, Jeon HJ. Anxiety symptoms are linked to new-onset suicidal ideation after six months of follow-up in outpatients with major depressive disorder. J Affect Disord 2015; 187:183-7.

3. Beghi M, Rosenbaum JF: Risk factors for fatal and nonfatal repetition of suicide attempt: a critical appraisal. Curr Opin Psychiatry 2010; 23:349-55.

4. Brière FN, Rohde P, Seeley JR, Klein D, Lewinsohn PM: Adolescent suicide attempts and adult adjustment. Depress Anxiety 2015; 32:270-6.

5. Capron DW, Allan NP, Ialongo NS, Leen-Feldner E, Schmidt NB: The depression distress amplification model in adolescents: A longitudinal examination of anxiety sensitivity cognitive concerns, depression and suicidal ideation. J Adolesc 2015; 41:17-24.

6. Capron DW, Cougle JR, Ribeiro JD, Joiner TE, Schmidt $N B$ : An interactive model of anxiety sensitivity relevant to suicide attempt history and future suicidal ideation. $J$ Psychiatr Res 2012b;46:174-80.

7. Capron WD, Blumental H, Medley AN, Lewis S, Feldner $M T$, Zvolensky MJ et al.: Anxiety sensitivity cognitive concerns predict suicidality among smokers. J Affect Disord 2012c;138:239-46.

8. Capron DW, Fitch K, Medley A, Blagg C, Mallott M, Joiner T: Role of anxiety sensitivity subfactors in suicidal ideation and suicide attempt history. Depress Anxiety 2012a;29:195-201.

9. Capron DW, Norr AM, Macatee RJ, Schmidt NB: Distress tolerance and anxiety sensitivity cognitive concerns: testing the incremental contributions of affect dysregulation constructs on suicidal ideation and suicide attempt. Behav Ther 2013; 44:349-58.

10. Cull JG \& Gill WS: Suicide Probability Scale (SPS) Manual. LA: Western Psychological Services, 1988.

11. Davidson RJ, Lewis DA, Alloy LB, Amaral DG, Bush G, Cohen JD, et al.: Neural and behavioral substrates of mood and mood regulation. Biol Psychiatry 2002: 52: 478-502.

12. Eskin M: Age specific suicide rates and the rates of increase, and suicide methods in Sweden and Turkey: A comparison of the official suicide statistics. Reports from the Department of Psychology, Stockholm University, No: 772, 1999.

13. Essau CA, Sasagawa S, Ollendick TH: The facets of anxiety sensitivity in adolescents. $J$ Anxiety Disord 2010;24: 23-9.

14. Foley D, Goldston D, Costello E, Angold A: Proximal psychiatric risk factors for suicidality in youth: the Great Smoky Mountains Study. Arch Gen Psychiatry 2006; 63:1017-1024.

15. Ghaziuddin N, King CA, Naylor MW, Ghaziuddin M: Anxiety contributes to suicidality in depressed adolescents. Depress Anxiety 2000;11:134-8.

16. Gokler B, Unal F, Pehlivanturk B, Cengel Kultur E, Devrim Akdemir D, Taner Y: Reliability and validity of Schedule for affective disorders and schizophrenia for school age children - present and lifetime version Turkish version (K-SADS-PL-T). Turk $J$ Child Adol Mental Healt 2004; 11:109-6 (in Turkish).

17. Gould MS, King R, Greenwald S, Fisher P, Schwab-Stone $M$, Kramer $R$, et al.: Psychopathology associated with suicidal ideation and attempts among children and adolescents. J Am Acad Child Adolesc Psychiatry 1998; 37:915-23.

18. Hu LT, Bentler PM: Cutoff criteria for fit indexes in covariance structure analysis: conventional criteria versus new alternatives. Struct Equ Model 1999; 6:1-55.

19. Joiner TE: Why people die by suicide. Cambridge, Mass.: Harvard University Press, 2005.

20. Katz C, Yaseen ZS, Mojtabai R, Cohen LJ, Galynker II: Panic as an independent risk factor for suicide attempt in depressive illness: findings from the National Epidemiological Survey on Alcohol and Related Conditions (NESARC). J Clin Psychiatry 2011;72:1628-35.

21. Kaufman J, Birmaher B, Brent D, Rao U, Flynn C, Moreci $P$ et al.: Schedule for affective disorders and schizophrenia for school-age children-present and lifetime version $(K-$ $S A D S-P L)$ : initial reliability and validity data. $J$ Am Acad Child Adolesc Psychiatry 1997;36:980-8.

22. Kovacs M: The Children's Depression Inventory (CDI). Psychopharmacol Bull 1985;21:995-8.

23. Lambert SF, Cooley MR, Campbell KD, Benoit MZ, Stansbury R: Assessing anxiety sensitivity in inner-city African American children: psychometric properties of the childhood anxiety sensitivity index. J Clin Child Adolesc Psychol 2004;33: 248-59.

24. Lamis DA, Jahn DR: Parent-child conflict and suicide rumination in college students: the mediating roles of depressive symptoms and anxiety sensitivity. J Am Coll Health 2013; 61:106-13.

25. Mathew AR, Pettit JW, Lewinsohn PM, Seeley JR, Roberts R: Co-morbidity between major depressive disorder and anxiety disorders: shared etiology or direct causation? Psychol Med 2011; 41:2023-34.

26. Maughan B, Collishaw S, Stringaris A: Depression in childhood and adolescence. J Can Acad Child Adolesc Psychiatry 2013; 22:35-40.

27. McLaughlin EN, Stewart SH, Taylor S: Childhood anxiety sensitivity index factors predict unique variance in DSM-IV anxiety disorder symptoms. Cogn Behav Ther 2007; 36:210-9.

28. Ocañez KL, Kathryn McHugh $R$, Otto MW: $A$ metaanalytic review of the association between anxiety sensitivity and pain. Depress Anxiety 2010;27:760-7. 
29. Öy B: Children's Depression Inventory: a study of reliability and validity. Turk $J$ Psychiatry 1991;2: 132-6 (In Turkish).

30. Reiss S, Peterson RA, Gursky DM, McNally RJ: Anxiety sensitivity, anxiety frequency and the predictions of fearfulness. Behav Res Ther 1986;24:1-8.

31. Sareen J, Cox BJ, Afifi TO, de Graaf R, Asmundson GJ, ten Have M, Stein MB: Anxiety disorders and risk for suicidal ideation and suicide attempts: A populationbased longitudinal study of adults. Arch Gen Psychiatry 2005;62:1249-57.

32. Schmidt NB, Woolaway-Bickel K, Bates M: Evaluating panic-specific factors in the relationship between suicide and panic disorder. Behav Res Ther 2001;39:635-49.

33. Schmidt NB, Zvolensky MJ, Maner JK: Anxiety sensitivity: prospective prediction of panic attacks and Axis I pathology. J Psychiatry Res 2006;40: 691-9.

34. Silverman $W K$, Fleisig $W$, Rabian B, Peterson RA: Childhood anxiety sensitivity index. J Clin Child Psychol 1991;20:162-8.

35. Tabachnick BG, Fidell LS: Using Multivariate Statistics, 5th edn. Allyn and Bacon, New York, 2007.

36. Vanderwerker L, Chen J, Charpentier P, Paulk ME, Michalski M, Prigerson HG: Differencs in risk factors for suicidality between African American and white patients vulnerable to suicide. Suicide Life Threat Behav 2007; 37:1-9.

37. Yllmaz S, Kllıç EZ: Adaptation of the Childhood Anxiety Sensitivity Index for Use in Turkey. Turk Psychiatry Derg, in press, 2014.

Correspondence:

Ayhan Bilgiç, MD

Associate Professor of Child and Adolescent Psychiatry

Necmettin Erbakan Üniversitesi, Meram Tıp Fakültesi, Çocuk ve Ergen Psikiyatrisi AD

42090, Meram / Konya, Turkey

E-mail:bilgicayhan@yahoo.com 